\title{
Efficient Capsid Antigen Presentation From Adeno-Associated Virus Empty Virions In Vivo
}

\author{
Xiaolei Pei $i^{1,2}$, Lauriel Freya Earley², Yi He ${ }^{1,2}$, Xiaojing Chen $^{2}$, Nikita Elexa Hall ${ }^{2}$, \\ Richard Jude Samulski ${ }^{2,3}$ and Chengwen $\mathrm{Li}^{2,4 *}$
}

${ }^{1}$ Chinese Academy of Medical Sciences and Peking Union Medical College, Institute of Hematology and Blood Diseases Hospital, Tianjin, China, ${ }^{2}$ Gene Therapy Center, University of North Carolina at Chapel Hill, Chapel Hill, NC, United States, ${ }^{3}$ Department of Pharmacology, University of North Carolina at Chapel Hill, Chapel Hill, NC, United States, ${ }^{4}$ Department of Pediatrics, University of North Carolina at Chapel Hill, Chapel Hill, NC, United States

Adeno-associated virus (AAV) vectors have been successfully applied in clinical trials for hemophilic patients. Although promising, the clinical results suggest that the capsid-specific CD8+T cell response has a negative effect on therapeutic success. In an in vitro analysis using an engineered AAV virus carrying immune-dominant SIINFEKL peptide in the capsid backbone, we have previously demonstrated that capsid antigen presentation from full (genome containing) AAV capsids requires endosome escape and is proteasome dependent

OPEN ACCESS

Edited by: Fabio Bagnoli, GlaxoSmithKline, Italy

Reviewed by: Arun Kumar, Linköping University, Sweden

Walter J. Storkus, University of Pittsburgh, United States

*Correspondence: Chengwen $L$ chengwen@med.unc.edu

Specialty section: This article was submitted to Vaccines and Molecular Therapeutics, a section of the journal Frontiers in Immunology

Received: 17 November 2017 Accepted: 05 April 2018 Published: 19 April 2018

Citation:

Pei X, Earley LF, He Y, Chen X, Hall NE, Samulski RJ and Li C (2018) Efficient Capsid Antigen Presentation From AdenoAssociated Virus

Empty Virions In Vivo.

Front. Immunol. 9:844. doi: 10.3389/fimmu.2018.00844 and that no capsid antigen presentation is induced from empty virions. In the present study, we examined capsid antigen presentation from administration of empty virions in animal models. In wild-type mice, similar to AAV full particles, capsid antigen presentation from AAV empty virion infection was dose dependent, and the kinetics studies showed that antigen presentation was detected from 2 to 40 days after AAV empty virion administration. In the transporter associated with antigen processing 1 deficient (TAP-/-) mice, capsid antigen presentation was inhibited from both AAV full and empty virions, but higher inhibition was achieved from AAV full particle administration than that from empty virions. This indicates that the pathway of capsid antigen presentation from AAV transduction is dependent on proteasome-mediated degradation of AAV capsids (mainly for full particles) and that the endosomal pathway may also play a role in antigen presentation from empty particles but not full virions. The capsid antigen presentation efficiency from AAV preparations was positively correlated with the amount of empty virions contaminated with full particles. Collectively, the results indicate that contamination of AAV empty virions induces efficient antigen presentation in vivo and the mechanism of capsid antigen presentation from empty virions involves both endosomal and proteasomal pathways. The elucidation of capsid antigen presentation from AAV empty virions may allow us to rationally design effective strategies to prevent elimination of AAV transduced target cells by capsid specific CD8+ T cells.

Keywords: adeno-associated virus, capsid, antigen presentation, CD8+ T cells, empty virions

\section{INTRODUCTION}

Adeno-associated virus (AAV) vectors have been successfully used to transduce hepatocytes in Phase I clinical trials in patients with hemophilia B (1-4). However, clinical results have suggested that capsid specific cytotoxic T lymphocytes (CTLs) eliminated AAV-transduced hepatocytes, which resulted in therapeutic failure (1-4). These observations pose an outstanding concern regarding 
capsid antigen presentation in AAV-transduced cells, which are recognized and eliminated by capsid specific CTLs in clinical trials. Most peptides loaded on MHC class I molecules are generated by proteasome degradation of newly synthesized ubiquitinated proteins, but exogenous proteins can also be presented on $\mathrm{MHC}$ class I molecules through cross-presentation. Two main intracellular pathways for cross-presentation have been described: endocytic (TAP and proteasome-independent) and cytosolic (TAP and proteasome-dependent) MHC class I peptide loading (5). In the TAP-independent pathway, exogenous antigens that have been endocytosed are degraded by proteases, and the resulting peptides bind to $\mathrm{MHC}$ class I molecules in late endosomes and lysosomes. In the TAP-dependent pathway, the internalized exogenous antigens are transferred from the endocytic pathway to the cytosol and degraded by proteasomes; the resulting peptides are then transported to the ER via TAP. Using pharmacological agents and AAV mutants, we have demonstrated that the classic MHC class I antigen presentation pathway plays a major role in AAV capsid antigen cross-presentation in AAV-transduced cells in vitro (6). However, the mechanism of capsid antigen crosspresentation from AAV-transduced cells in vivo is perhaps different from that in vitro due to the far more complex environment.

Adeno-associated virus vectors purified from cesium chloride ( $\mathrm{CsCl})$ density gradients have been applied in clinical trials; however, this purification approach is not scalable. Recently, ionexchange chromatography has been studied to purify AAV vectors (7-10). Unlike the $\mathrm{CsCl}$ approach, the chromatographic method cannot separate genome-containing particles of AAV vectors (full particles) from empty virions as it relies on the charge of the capsid surface. The contamination of vector preparations by empty virions may inhibit transduction of genome containing AAV vectors and potentially increases the virus capsid antigen load in transduced cells as dose is determined by genome copy number, not by total number of capsids (10). Although empty AAV particles contain the identical protein components required for trafficking as that in full particles, our preliminary results demonstrated that capsid antigen presentation was significantly reduced in AAV transduced cells infected with AAV2 empty virions compared to full particles in vitro (6). This phenomenon may be interpreted as insufficient escape of these empty virions from the endosome (11). Recently, empty AAV capsids were proposed to function as decoys in clinical trials to allow AAV full particles to escape neutralizing antibodies (12). However, liver damage was observed following systemic administration of AAV preparations contaminated with empty particles (13), which alludes to the possibility that the contamination of empty particles leads to increased capsid antigen presentation due to an increase in the number of capsids infused into the patients. In this study, our in vivo results demonstrate that capsid antigen presentation was induced from AAV empty virions. The capsid antigen presentation from empty and full AAV particles was dose dependent and occurred at an early time period after administration for AAV empty particles when compared to full particles. Contrary to AAV full particles, the capsid antigen presentation from empty virions may involve both antigen presentation pathways: endosomal dependent and proteasomal dependent. More efficient antigen presentation was observed when a high amount of empty virions were included in AAV preparations.

\section{MATERIALS AND METHODS}

\section{Cells and Virus}

HEK293 cells were maintained at $37^{\circ} \mathrm{C}$ and $5 \% \mathrm{CO}_{2}$ in Dulbecco's modified Eagle's medium with supplementation of $10 \%$ heatinactivated fetal calf serum, $100 \mathrm{U} / \mathrm{ml}$ penicillin, and $100 \mathrm{~g} / \mathrm{ml}$ streptomycin. AAV full particle virus production was previously described using three plasmid cotransfection in 293 cells (14). AAV empty virions were produced by transfection of 293 cells with the AAV-OVA helper and Ad helper plasmids without AAV transgene cassette plasmid and then purified by $\mathrm{CsCl}$ gradient centrifugation. The titer of the produced empty particles was determined using a Western blot with antibodies specifically detecting intact virions (A20 and ADK8 antibodies for AAV2 and AAV8, respectively). The full particle virus titer was determined by Southern dot blot. The denatured empty virions were verified by Western blot with A20 or B1 antibody. 293/H-2Kb cells are a derivative of the 293 cell line stably expressing mouse MHC class $\mathrm{I}(\mathrm{H}-2 \mathrm{~Kb})$.

\section{Mice}

C57BL/6 mice, TAP-/- mice which are unable to present cytosolic antigens to class I-restricted cytotoxic T cells by delivery of peptides across the endoplasmic reticulum membrane to class I molecules, and OT-1/Rag-1 mice with expression of an $\mathrm{H} 2 \mathrm{~Kb}$ restricted $\mathrm{T}$-cell receptor specific for the OVA-derived SIINFEKL peptide were purchased from Jackson Laboratory (Bar Harbor, ME, USA). All mice were maintained in a specific pathogen-free facility at the University of North Carolina at Chapel Hill. The University of North Carolina Institutional Animal Care and Use Committee approved all procedures.

\section{Enzyme-Linked Immunosorbent Assay (ELISA) for Human Alpha- 1-Antitrypsin (AAT)}

Alpha-1-antitrypsin concentration in blood was carried out using an ELISA as described previously (15).

\section{In Vivo T Cell Proliferation}

AAVOVA vector was injected in C57BL/6 mice or TAP-/mice via retro-orbital administration. Spleen cells were collected from OT-1 mice and labeled with CFSE, then transfused into C57BL/6 mice or TAP-/- mice after AAV administration. At indicated time following OT-1 T cell transfer, spleen cells were harvested, and the frequency of $\mathrm{T}$ cells in proliferation status was determined by flow cytometer (16). Consistent with our previous study, majority of OT-1 spleen cells in proliferation were CD8+ $\mathrm{T}$ cells in vitro and in vivo after AAV/OVA capsids were applied (Figure S1 in Supplementary Material). No significant CD4+ cell proliferation was observed in these studies, so we chose to perform proliferation analysis using whole spleen cells.

\section{Statistical Analysis}

The Student's $t$-test was used to perform statistical analysis. $P<0.05$ was considered a statistically significant difference. 


\section{RESULTS}

\section{AAV2 Empty Virions Induce Efficient Capsid Antigen Presentation In Vivo}

In our previous studies, we used an engineered AAV2 capsid by swapping the immune-domain epitope, SIINFEKL, from ovalbumin protein into the AAV virion HI loop and demonstrated that capsid antigen presentation is elicited from AAV2-transduced liver cells in mice (17). In reality, empty particles are impossible to completely remove from the AAV virus preparation, especially in AAV virion purification using column affinity. This raises a concern as to whether the contamination of empty virions in AAV preparations increases capsid antigen presentation in transduced cells. To evaluate this, we injected $2 \times 10^{11}$ particles of AAV2OVA full capsids or empty virions into C57BL/6 mice via systemic administration and 3 days later, CFSE conjugated OT-1 spleen cells were infused into the mice. At day 10 post OT- 1 cell transfusion, the mice were sacrificed, and spleen cells were collected for OT-1 spleen cell proliferation analysis. Surprisingly, $80 \%$ of OT- 1 cells were in division status in mice receiving AAV2OVA empty virions, a slightly higher value than that observed in mice with AAV2OVA full particle application (Figure 1, $p<0.05$ ). This result indicates that empty virions also induce capsid antigen presentation in vivo.

\section{Similar Kinetics of Capsid Antigen Presentation Between Full Particles and Empty Virions}

Our previous study has demonstrated that the efficiency of capsid antigen presentation from full particle transduction gradually decreases over time, and higher capsid antigen presentation

A
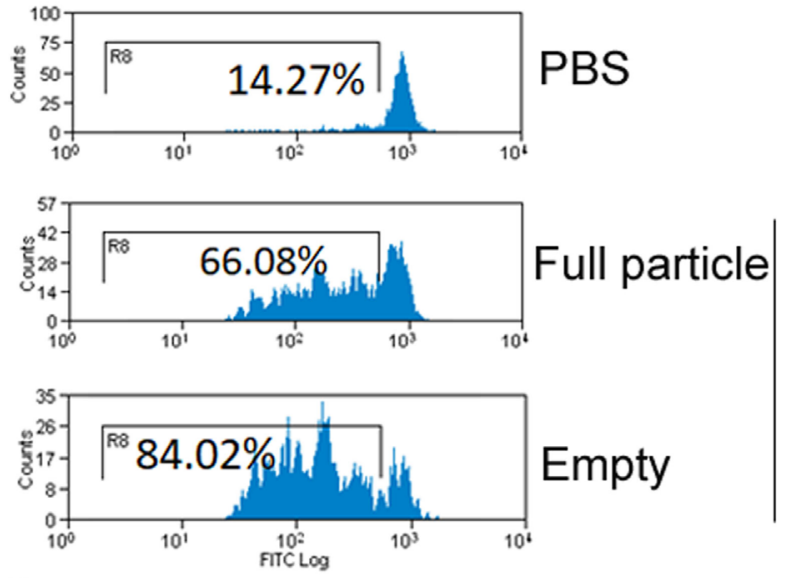

AAV2OVA

B $2 \times 10^{11}$ particles

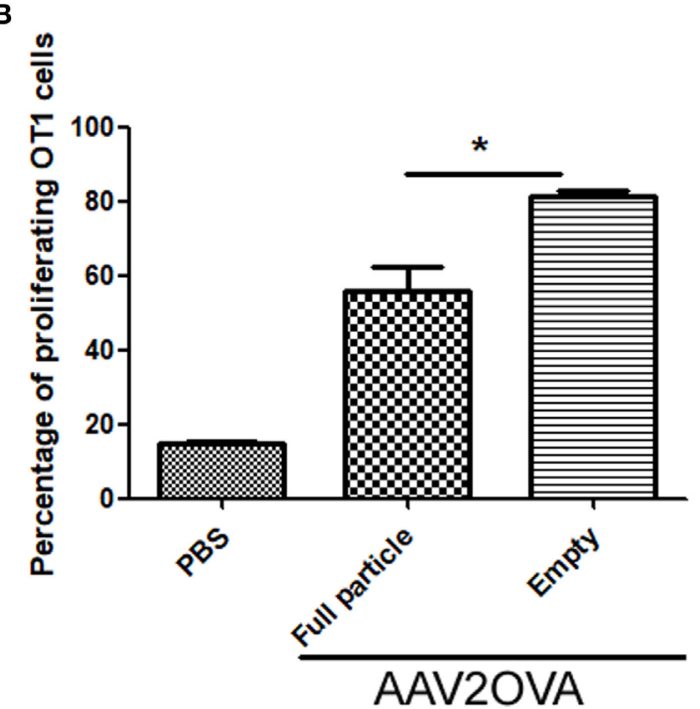

FIGURE 1 | Efficient capsid antigen presentation from systemic administration of AAV2OVA empty virions in mice. $2 \times 10^{11}$ particles of AAV2OVA empty virions or full particle vectors encoding transgene alpha-1-antitrypsin were intravenously injected into C57BL/6 mice via retro-orbital vein. At day 3 post adeno-associated virus (AAV) injection, $5 \times 10^{6} \mathrm{CFSE}-$ labeled OT-1 T cells were transferred into the mice. Ten days after transfer of OT-1 cells, the proliferation of OT-1 T cells was measured by flow cytometry. (A) Representative flow cytometric histograms. (B) Average T cell proliferation and SD for four mice. ${ }^{*} p<0.05$ compared to mice with AAV full particle treatment. 
occurs at an early time after AAV transduction in vivo (18). Next, we examined whether capsid antigen presentation from empty virion infection followed a similar trend to that of full particles. We injected $2 \times 10^{11}$ particles of AAV2OVA empty virions into C57BL/ 6 mice, and at days 3, 20, and 30 post AAV administration, CFSE-conjugated OT-1 spleen cells were transfused. Ten days later post OT-1 injection, the efficiency of antigen presentation was analyzed. As shown in Figure 2, capsid antigen presentation was much lower when OT-1 cells were infused at days 20 and 30 than that when OT-1 cells were infused at day 3 post AAV empty virion application. No antigen presentation was observed at days 30 and 40 with OT-1 injection. These results indicate a similar kinetics of capsid antigen presentation between full particles and empty particle. In our previous study, we demonstrated that no capsid antigen presentation was observed in $293 / \mathrm{H}-2 \mathrm{~Kb}$ cells infected with AAV2OVA empty virions in vitro (6). This prior experiment was performed within a short time of both AAV transduction $(<48 \mathrm{~h})$ and of incubation of AAV transduced cells with OT-1 spleens $(<24 \mathrm{~h})$, which is different from the latest experiment carried out with a relative long time of transduction and of interaction of AAV transduced cells with OT-1 spleen cells in vivo. To investigate the capsid antigen presentation efficiency from empty virions immediately followed by AAV infection in vivo, OT-1 spleen cells were infused at day 1 after AAV systemic administration, and antigen presentation was assayed at days 1, 2, and 3 post OT-1 cell injection. No capsid antigen presentation was detected at day 1 post OT-1 infusion, similar to the in vitro finding for capsid antigen presentation

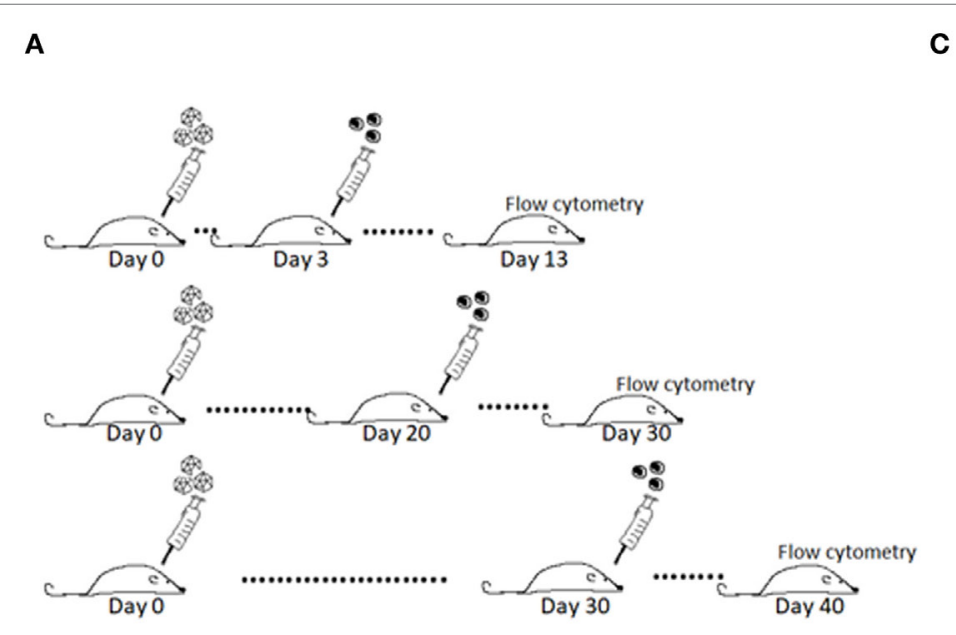

B

Day 3-13

Day 20-30
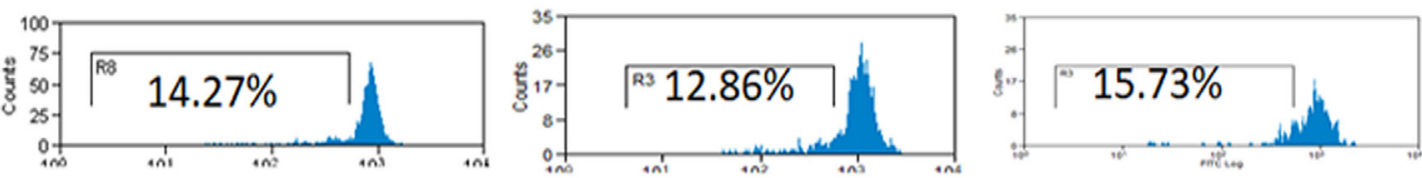

PBS
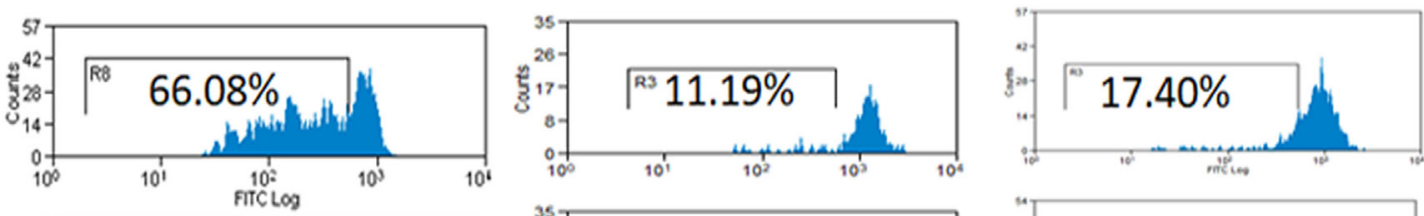

Full particle
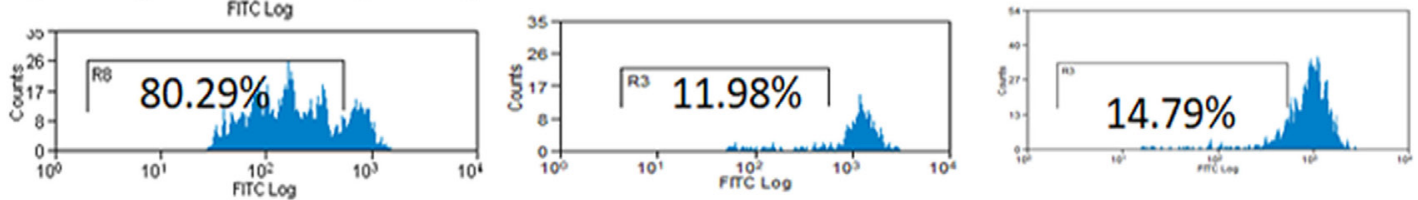

Empty

FIGURE 2 | The kinetics of capsid antigen presentation from AAV2OVA empty virions in mice. AAVOVA viruses (empty or full) at a dose of $2 \times 10^{11}$ were systemically administered via retro-orbital injection into C57BL/6 mice, and at the indicated time points, CFSE-labeled OT-1 T cells were transfused. At day 10 post OT-1 infusion, the proliferation of OT-1 T cells was analyzed by flow cytometry. (A) A scheme for time points of adeno-associated virus administration and OT-1 cells injection as well as assay for OT-1 spleen cell proliferation. (B) Representative flow cytometric histograms. (C) Average T cell proliferation and SD for four mice. 
from empty virions, but contrary to that from full particles. Comparable capsid antigen presentation was demonstrated in days 2 and 3 post OT- 1 cell transfer in mice receiving either full particles or empty virions (Figure 3). These results suggest that capsid antigen presentation occurs at an early phase after virion infection.

\section{Capsid Antigen Presentation From AAV2 Empty Virions Is Dose Dependent In Vivo}

To study dose-dependent capsid antigen presentation, we injected mice with different doses of AAV2OVA empty virions and infused OT-1 spleen cells 3 days later. Capsid antigen presentation was analyzed at day 10 post OT-1 infusion. As shown in Figure 4, when $1 \times 10^{10}$ empty virions were administered, we did not detect any capsid antigen presentation. However, when $5 \times 10^{10}$ empty virions were injected, capsid antigen presentation was observed. This result is consistent with the observation from capsid antigen presentation using AAV2 full particles in our previous report, in which antigen presentation is dose dependent (18).

\section{Capsid Antigen Presentation From AAV8 Empty Virions In Vivo}

To study whether capsid antigen presentation from empty virions also applied to other serotypes, we made AAV8OVA empty virions. After injection of $2 \times 10^{11}$ AAV8OVA empty particles, OT-1 spleen cells were transfused at day 3 and 10 days later, the mice were sacrificed, and spleen cells were collected for an OT-1 spleen cell proliferation assay. As shown in Figure 5,
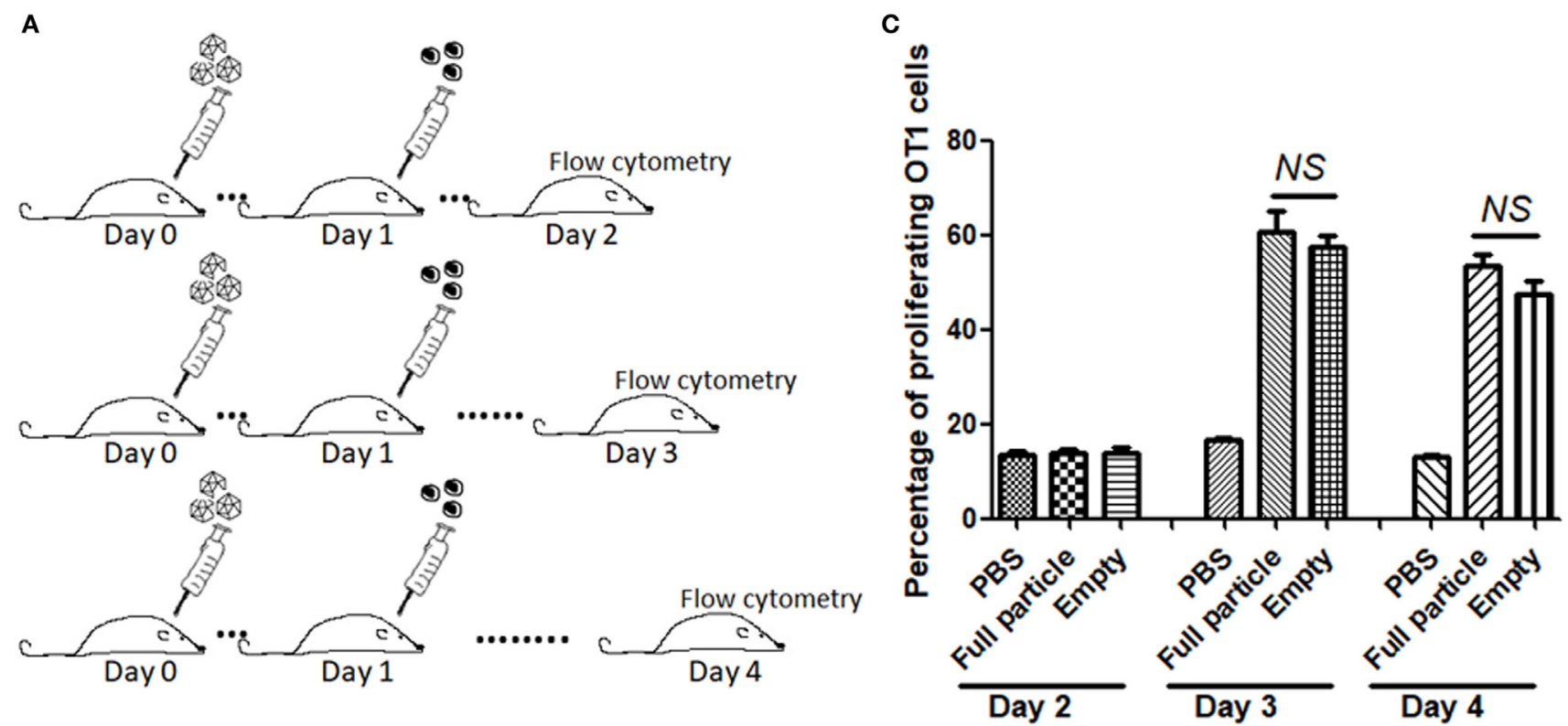

B

Day 2

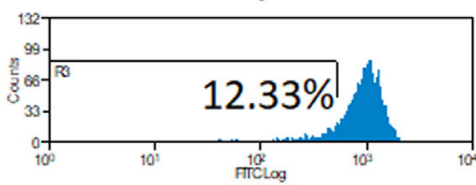

Day 3
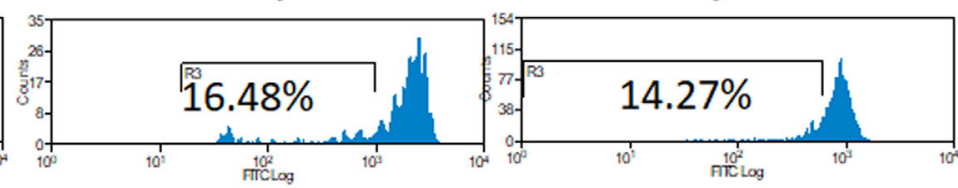

PBS
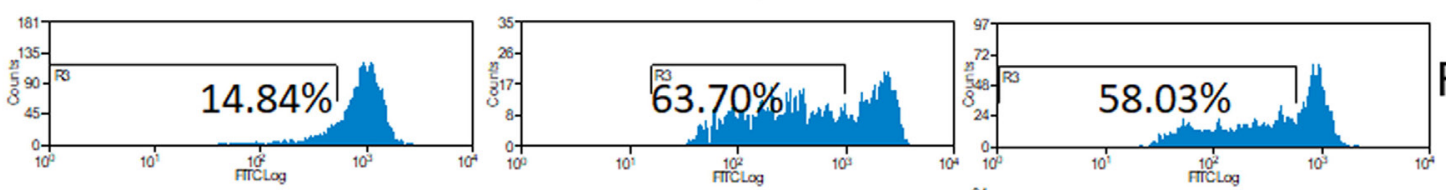

Full particle
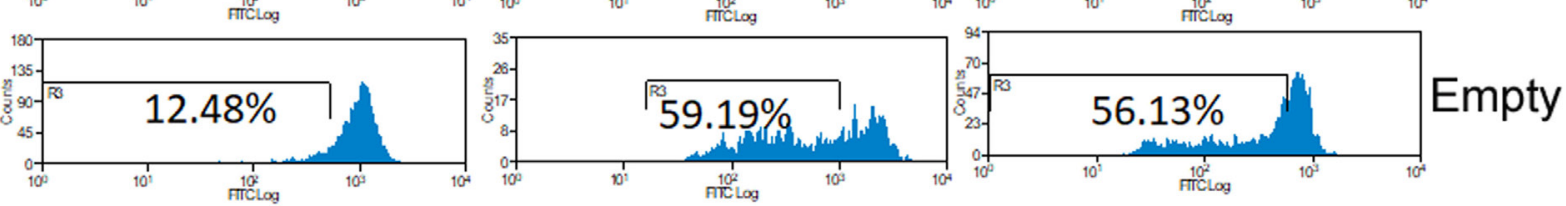

FIGURE 3 | Capsid antigen presentation from AAV2OVA empty virions at early time points after administration in mice. $2 \times 10^{11}$ particles of AAVOVA viruses were systemically injected via retro-orbital vein into C57BL/6 mice. One day later, CFSE-labeled OT-1 spleen cells were transferred into the mice. At days 1 , 2, and 3 post OT-1 cell infusion, the proliferation of OT-1 cells was analyzed by flow cytometry. (A) A scheme for time points of adeno-associated virus administration and OT-1 cells injection as well as assay for OT-1 spleen cell proliferation. (B) Representative flow cytometric histograms. (C) Average T cell proliferation and SD for four mice. 
A
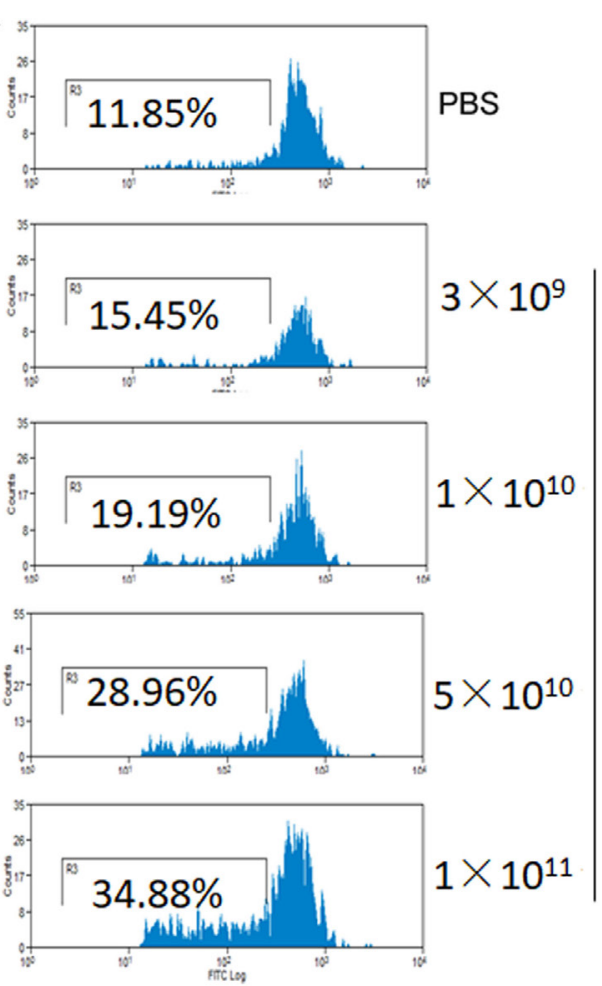

PBS

$3 \times 10^{9}$

$5 \times 10^{10}$
B

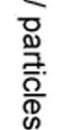
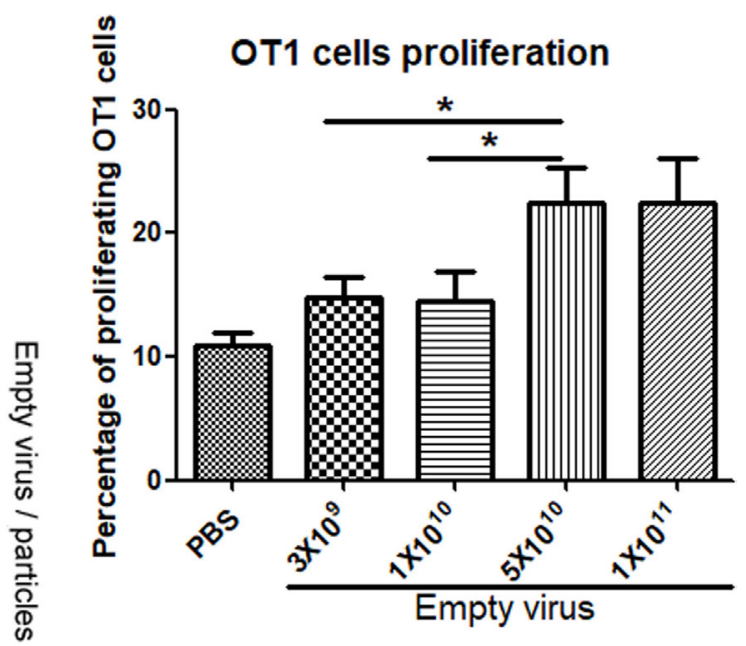

FIGURE 4 | Capsid antigen presentation from AAV2OVA empty virions is dose responsive in vivo. Different doses of AAV2OVA empty vectors were intravenously injected into C57BL/6 mice and 3 days later, and CFSE-labeled OT-1 T cells were transferred into the mice. At day 10 post injection of OT-1 cells, T cell proliferation in the spleen was analyzed via flow cytometry. (A) Representative flow cytometric histograms. (B) Average T cell proliferation and SD of five mice. ${ }^{\star} p<0.05$ when compared to mice with the low dose of AAV treatment.

$\mathbf{A}_{12}$
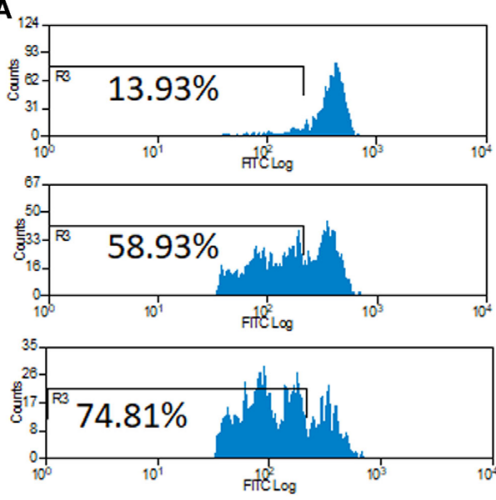

PBS

Empty
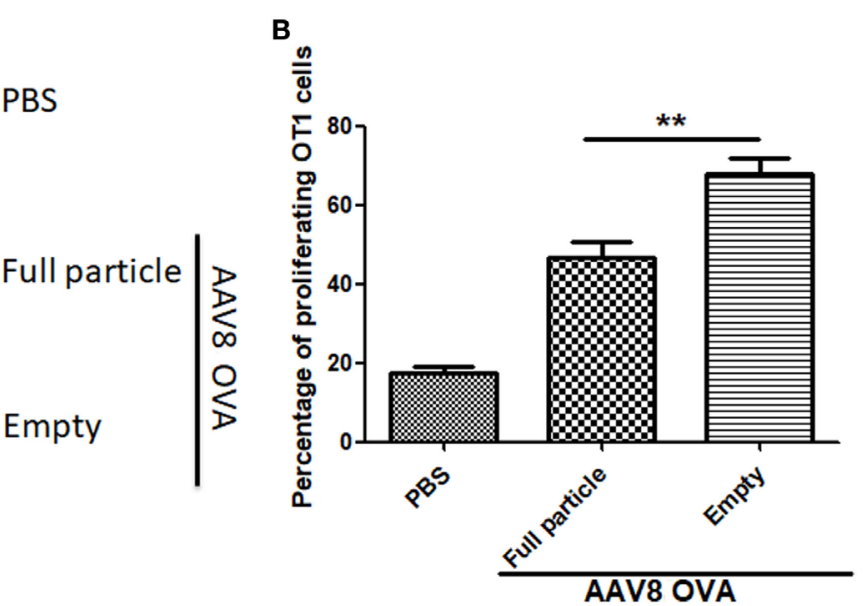

FIGURE 5 | The capsid antigen presentation from AAV8OVA empty virions in mice. $2 \times 10^{11}$ particles of AAV8OVA viruses (empty or full) were injected into C57BL/6 mice via retro-orbital vein, and at day 3 post AAV8OVA injection, $5 \times 10^{6}$ CFSE-labeled OT-1 T cells were transferred. Ten days posttransfer, proliferation of OT-1 cells was measured by flow cytometry. (A) Representative flow cytometric histograms. (B) Average T cell proliferation and SD for four mice. ${ }^{\star} p<0.05$ when compared to mice treated with AAV8OVA full particles.

similar to capsid antigen presentation from AAV2 empty virions, efficient capsid antigen presentation from AAV8 empty virions was also detected and was higher than that from AAV8 full particles. These data implicate that capsid antigen presentation from empty virions can likely be induced from any AAV serotype in vivo. 


\section{Contamination of Empty Virions in AAV Preparation Increases Capsid Antigen Presentation}

To study the contribution of empty virions in AAV preparation to capsid antigen presentation, we mixed different amounts of empty virions into $1 \times 10^{11}$ particles of AAV full particles of AAV2OVA capsids containing a CBA-driven AAT genome. At day 3 after injection of the mixture of AAV full particles with empty virions into mice, OT-1 spleen cells were infused and 10 days later the spleen cells were harvested for antigen presentation analysis. As described earlier, capsid antigen presentation was detected when $1 \times 10^{11}$ particles of full particles only were injected, and significantly increased when empty virions were added (Figure 6). This study supports the hypothesis that the contamination of empty virions in AAV preparation increases AAV antigen load, which may induce higher elimination of AAV-transduced target cells mediated by capsid-specific CTLs. To examine whether the contamination of empty virions had an impact on AAV transduction from full particles, blood was collected at day 13 post AAV injection when mice were sacrificed for capsid antigen presentation analysis, and transgene AAT expression was measured by ELISA. As shown in Figure 7, the AAT level in blood was similar among the different groups regardless of the amount of empty virions added to the fixed dose of AAV2 full particles.

\section{Blockage of Proteasome Pathway Partially Inhibits Capsid Antigen Presentation From Empty Capsids}

In our previous study, we demonstrated that capsid antigen presentation from AAV-transduced cells is proteasome dependent and requires endosomal escape (6). Utilization of proteasome inhibitors completely blocks capsid antigen presentation in $293 / \mathrm{H}-2 \mathrm{~Kb}$ cells in a short incubation period. To study which antigen presentation pathway is involved in capsid antigen presentation in vivo after AAV transduction, TAP - / - mice were chosen since TAP deficiency blocks antigen presentation from proteasome-mediated degradation. $1 \times 10^{11}$ particles of AAV2OVA full particles were administered into TAP-/ - mice and wild-type C57BL/ 6 mice. At day 3 post AAV injection, OT-1 cells were transfused into the mice and 10 days later, spleen cells were collected for a capsid antigen presentation assay. Consistent to the finding in $293 / \mathrm{H}-2 \mathrm{~Kb}$ cells, no capsid antigen presentation was documented in $\mathrm{TAP}-/-$ mice, although significantly higher capsid antigen presentation was observed in wild-type mice receiving AAV2OVA full particles when compared to control mice only receiving PBS injection (Figure 8). When $1 \times 10^{11}$ particles of AAV2OVA virions were administered in TAP $-/-$ mice, there was no significant difference in OT1 cell proliferation between mice injected with PBS or full particles; however, capsid antigen presentation from
A

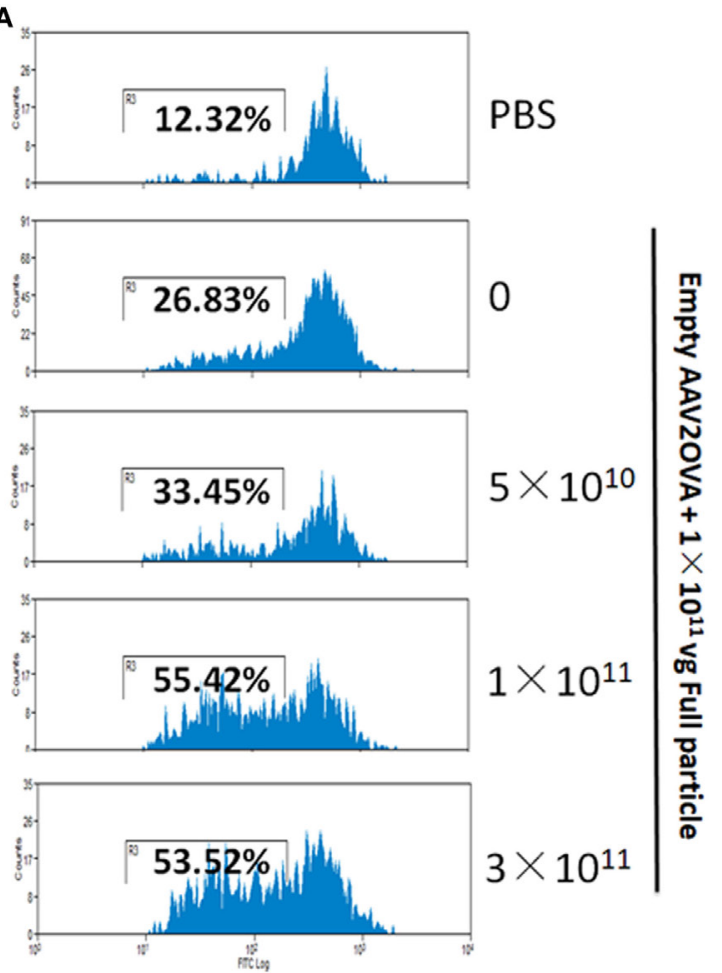

B

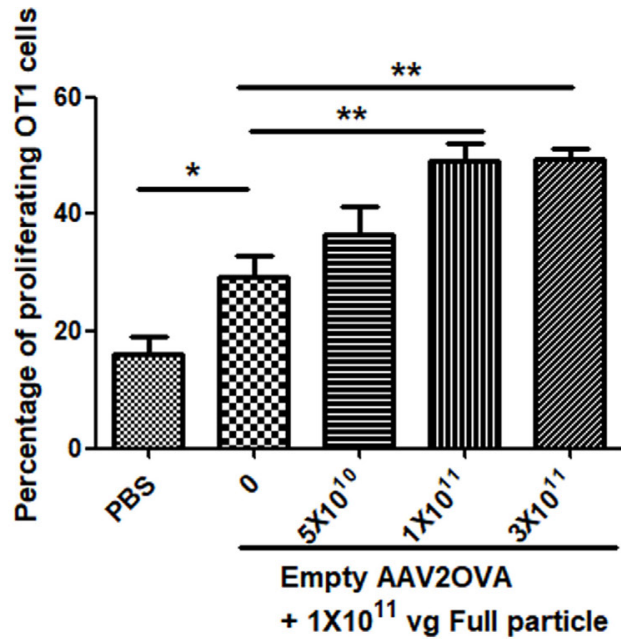

FIGURE 6 | Contamination of empty virions in adeno-associated virus (AAV) preparation increases capsid antigen presentation. Different amounts of AAV2OVA empty virions were mixed with $1 \times 10^{11}$ particles of AAV2OVAValpha-1-antitrypsin (AAT) vectors and then administered into C57BL mice via retro-orbital injection. At day 3 post AAV injection, OT-1 spleen cells were transfused. Ten days later, mice were killed, and spleen cells were collected for OT-1 cell proliferation assay. (A) Representative flow cytometric histograms. (B) Average T cell proliferation and SD from five mice. 
empty capsids was higher than that of AAV2OVA full particles. This result suggests that the capsid antigen presentation from AAV empty virions may use different mechanisms to process antigens, and this process is less dependent on TAP antigen presentation. It was noted that OT-1 cell proliferation in TAP $-/-$ control mice was higher than that in wild-type C57BL/6 mice, the difference may result from the different immune background of mouse strains.

\section{DISCUSSION}

In this study, we demonstrated that efficient capsid antigen presentation, as measured by $\mathrm{CD} 8+\mathrm{T}$-cell proliferation, was

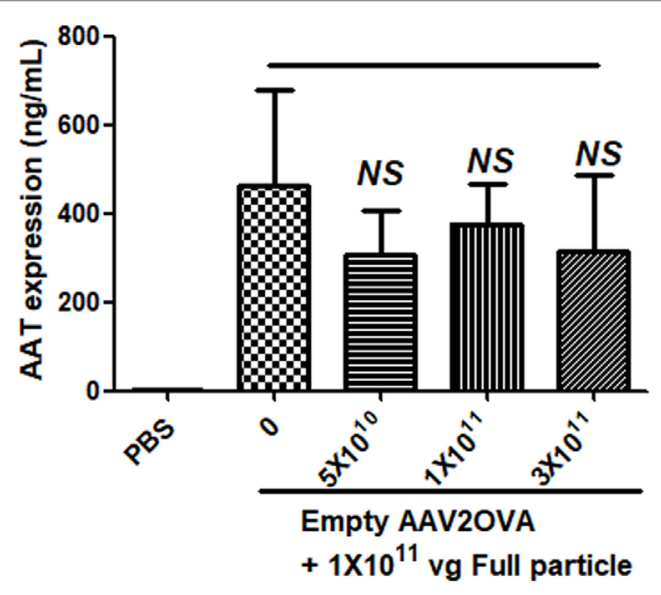

FIGURE 7 | No effect of empty virions on transgene expression from adeno-associated virus full particles after systemic administration. Blood was collected from mice in Figure $\mathbf{6}$ when mice were sacrificed for capsid antigen presentation analysis. Transgene alpha-1-antitrypsin (AAT) expression was detected by enzyme-linked immunosorbent assay. The result is represented as the average and SD of five mice. achieved from administration of AAV empty virions in wildtype mice. In agreement with the capsid antigen presentation from AAV full particles in mice, the capsid antigen presentation from AAV2 empty particles was dose dependent and detected with higher presentation than full particles at the early phase of AAV application. While the TAP pathway plays a major role in capsid antigen presentation from AAV full particles, both TAP and endosomal pathways may be involved in the capsid antigen presentation from empty virions in vivo. Contamination of empty virions in AAV preparation resulted in higher amounts of T-cell proliferation, indicating higher amounts of capsid antigen presentation.

Our previous studies have demonstrated that AAV full particles induce efficient capsid antigen presentation in mice and our mechanism study showed that capsid antigen presentation is proteasomal dependent in an in vitro well-defined cell line $(6,18)$; however, the capsid antigen presentation was not detected from empty virions in vitro (6). These results raise the question as to how capsid antigen presentation occurs from AAV empty virions in vivo. In considering this, the following factors may be important: (1) different environments between AAV transduced cells in vivo and in vitro including cell-cell interactions or cells with components around cells, (2) intracellular trafficking, and (3) the long-term interaction of AAV virions with cellular proteins. These factors likely help to explain the inconsistent results obtained from the in vitro assay, in which no detectable capsid antigen presentation was observed in cells infected with AAV empty virions at $48 \mathrm{~h}$ post infection, and the in vivo administration of empty virions which induced capsid antigen presentation similar to that of AAV full particles in mice. There was a trend that capsid antigen presentation was higher in mice treated with AAV full particles before day 4 of transduction than that in mice receiving empty virions. After day 4 , the capsid antigen presentation was more prominent in mice treated with empty virions
A
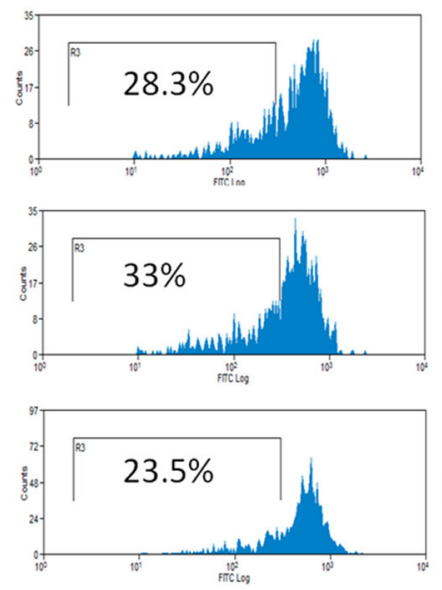

PBS

Full particle

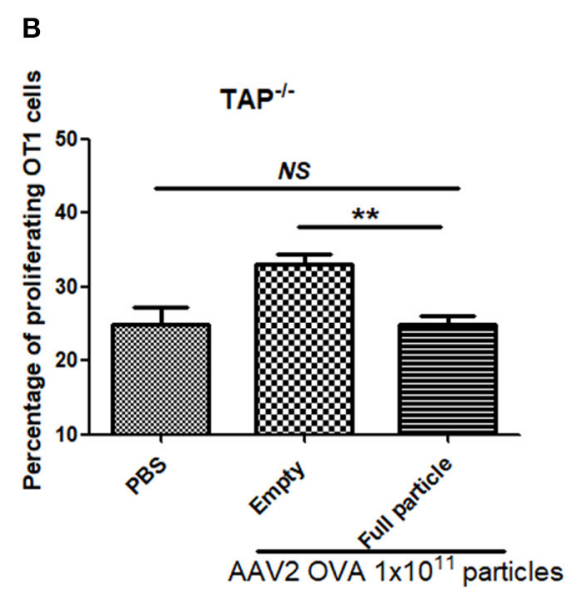

FIGURE 8 | Blockage of proteasome pathway inhibits capsid antigen presentation from empty capsid. $1 \times 10^{11}$ particles of AAV2OVA vectors (empty or full) were intravenously injected into TAP-/- mice via retro-orbital vein. At day 3, OT-1 cells were administered into the mice. Ten days later, spleen cells from mice were harvested for capsid antigen presentation analysis. (A) Representative flow cytometric histograms in TAP-/- mice treated with adeno-associated virus particles. (B) Average T cell proliferation and SD for five mice. ${ }^{* \star} p<0.01$ when compared to mice treated with AAV2OVA full particles in TAP-/- mice. 
than that with full particles. These results indicate that (1) capsid antigen presentation from full particles may occur faster than that from empty virions in vivo, which suggests a different mechanism of capsid antigen presentation between AAV full particles and empty virions and (2) the in vitro assay was not performed at a sufficiently long enough time point to demonstrate antigen presentation from empty capsids.

Using pharmacological agents and AAV mutants in an in vitro analysis, we have previously demonstrated that capsid antigen presentation from AAV full particles requires efficient endosomal escape into the cytosol and capsid degradation by proteasomes, supporting the hypothesis that the proteasomal pathway is involved in capsid antigen presentation from AAV-transduced cells (6). In the present study, we compared the efficiency of capsid antigen presentation induced from full particles or empty virions in wild-type mice and mice with TAP deficiency, in which antigen presentation from proteasomemediated degradation of antigen is blocked. Efficient capsid antigen presentation was detected in wild-type mice treated with either full particles or empty virions. When compared to control TAP-/- mice without AAV administration, no increased capsid antigen presentation was observed in TAP-/- mice treated with either full particles or empty virions, instead, we observed a reduction in capsid antigen presentation for the full particles. This result implicates that both AAV full and empty capsids are capable of inducing capsid antigen presentation via the cytosolic pathway. However, it should be noted that a little higher capsid antigen presentation was detected in TAP-/mice treated with empty virions than that with full particles, suggesting that the endosomal pathway may also be involved in capsid antigen presentation from empty virions. These results, based on the kinetics of capsid antigen presentation and the mechanism studies of capsid antigen presentation from AAV full particles and empty virions in vivo, are supported by the findings that AAV full particles can escape from endosomes and traffic to the nucleus more efficiently than empty virions (19). Although both full and empty virions contain VP1, which harbors both the phospholipase A2 (PLA2) domain and nuclear localization signals (NLSs) required for efficient intracellular trafficking $(11,20,21)$, empty particles are deficient in exposure of the N-terminal of VP1/2 of AAV virions when compared to full particles (22).

One interesting finding from the present study is that efficient capsid antigen presentation was achieved from denatured AAV virions. Although we did not perform a mechanism study, we presume that the endosomal pathway would be involved due to the fact that most denatured capsids should be VP3 subunits since VP3 accounts for $80 \%$ VP subunits and does not have the PLA2 domain and NLSs on its N-terminal $(20,21)$. This finding also implicates that elimination of denatured AAV capsid subunits in AAV preparation has the potential to decrease AAV capsid antigen load.

It has been reported that the contamination of empty virions inhibits the transduction from AAV full particles after muscular injection (10). In this study, we did not observe the inhibition effect of contaminated empty virions on AAV transduction from full capsids after systemic administration, similar to the observation from Mingozzi et al. (12). This difference can be attributed to variable administration routes and different targeted tissues. When AAV vectors were intramuscularly administered, the majority of AAV viruses accumulated around the injection site, and the ratio of AAV particles per muscle fiber was very high. Therefore, the high amount of empty virions competed with full particles to bind to the muscle surface and inhibited transduction from full particles. However, after systemic administration, AAV viruses circulated in the blood and were bound to cells in every tissue of the body. Although high doses of AAV vectors, including empty virions and full particles, are used for systemic injection, the AAV capsids per targeted cell would likely have been lower compared to muscle injection and the empty virions were not concentrated enough to compete with full particles for cell binding and intracellular trafficking, so there is no inhibition effect of empty virions on transgene expression from AAV full particles using IV injection. It is expected that transgene expression will be decreased when an extra high dose of empty virions is used.

In conclusion, our study demonstrates that efficient capsid antigen presentation can be induced from AAV empty capsids. The efficiency of the capsid antigen presentation from AAV empty virions is dose dependent and occurs in the early periods after administration of empty virions. The proteasome pathway plays a role in capsid antigen presentation from both full and empty particles, but the endosomal pathway may also be involved in capsid antigen presentation from empty virions. Contamination of empty virions increases capsid antigen presentation from AAV transduction. In summary, studies on the capsid antigen presentation from AAV empty virions in vivo will allow us to design effective approaches to prevent and block capsid-specific CTL-mediated elimination of AAV transduced target cells in future clinical trials.

\section{ETHICS STATEMENT}

This study was carried out in accordance with the recommendations of "University Research Policies and Procedures, Institutional Animal Care and Use Committee at UNC". The protocol was approved by the Institutional Animal Care and Use Committee at UNC.

\section{AUTHOR CONTRIBUTIONS}

XP completed the majority of this research including Figures 1-8 and participated in project design. YH completed the experiment including Figure 2, XC worked as the technician to provide experiment skill support, and RS and CL designed the project.

\section{FUNDING}

This work was supported by National Institutes of Health Grants R01AI117408 (to CL), R01HL125749 (to CL), R01AI072176, and R01AR064369 (to RS). Animal Studies were performed within the LCCC Animal Studies Core Facility at the University of North Carolina at Chapel Hill. The LCCC Animal Studies 
Core is supported in part by an NCI Center Core Support Grant (CA16086) to the UNC Lineberger Comprehensive Cancer Center. The UNC Flow Cytometry Core Facility is supported in part by an NCI Center Core Support Grant (P30CA06086) to the UNC Lineberger Comprehensive Cancer Center.

\section{REFERENCES}

1. Manno CS, Pierce GF, Arruda VR, Glader B, Ragni M, Rasko JJ, et al. Successful transduction of liver in hemophilia by AAV-Factor IX and limitations imposed by the host immune response. Nat Med (2006) 12:342-7. doi: $10.1038 / \mathrm{nm} 1358$

2. Nathwani AC, Tuddenham EG, Rangarajan S, Rosales C, McIntosh J, Linch DC, et al. Adenovirus-associated virus vector-mediated gene transfer in hemophilia B. NEngl J Med(2011) 365:2357-65. doi:10.1056/NEJMoa1108046

3. Nathwani AC, Reiss UM, Tuddenham EG, Rosales C, Chowdary P, McIntosh J, et al. Long-term safety and efficacy of factor IX gene therapy in hemophilia B. N Engl J Med (2014) 371:1994-2004. doi:10.1056/NEJMoa1407309

4. Nathwani AC, Davidoff AM, Tuddenham EGD. Advances in gene therapy for haemophilia. Hum Gene Ther (2017) 28(11):1004-12. doi:10.1089/ hum.2017.167

5. Inaba $\mathrm{K}$, Inaba $\mathrm{M}$. Antigen recognition and presentation by dendritic cells. Int J Hematol (2005) 81:181-7. doi:10.1532/IJH97.04200

6. Li C, He Y, Nicolson S, Hirsch M, Weinberg MS, Zhang P, et al. Adenoassociated virus capsid antigen presentation is dependent on endosomal escape. J Clin Invest (2013) 123:1390-401. doi:10.1172/JCI66611

7. Gao G, Qu G, Burnham MS, Huang J, Chirmule N, Joshi B, et al. Purification of recombinant adeno-associated virus vectors by column chromatography and its performance in vivo. Hum Gene Ther (2000) 11:2079-91. doi:10.1089/ 104303400750001390

8. Lock M, Alvira MR, Wilson JM. Analysis of particle content of recombinant adeno-associated virus serotype 8 vectors by ion-exchange chromatography. Hum Gene Ther Methods (2012) 23:56-64. doi:10.1089/hgtb.2011.217

9. Qu G, Bahr-Davidson J, Prado J, Tai A, Cataniag F, McDonnell J, et al. Separation of adeno-associated virus type 2 empty particles from genome containing vectors by anion-exchange column chromatography. JVirol Methods (2007) 140:183-92. doi:10.1016/j.jviromet.2006.11.019

10. Urabe M, Xin KQ, Obara Y, Nakakura T, Mizukami H, Kume A, et al. Removal of empty capsids from type 1 adeno-associated virus vector stocks by anion-exchange chromatography potentiates transgene expression. Mol Ther (2006) 13:823-8. doi:10.1016/j.ymthe.2006.08.1217

11. Johnson JS, Li C, DiPrimio N, Weinberg MS, McCown TJ, Samulski RJ. Mutagenesis of adeno-associated virus type 2 capsid protein VP1 uncovers new roles for basic amino acids in trafficking and cell-specific transduction. J Virol (2010) 84:8888-902. doi:10.1128/JVI.00687-10

12. Mingozzi F, Anguela XM, Pavani G, Chen Y, Davidson RJ, Hui DJ, et al. Overcoming preexisting humoral immunity to AAV using capsid decoys. Sci Transl Med (2013) 5:194ra192. doi:10.1126/scitranslmed.3005795

13. Gao K, Li M, Zhong L, Su Q, Li J, Li S, et al. Empty virions in AAV8 vector preparations reduce transduction efficiency and may cause total viral particle dose-limiting side effects. Mol Ther Methods Clin Dev (2014) 1:20139. doi: $10.1038 / \mathrm{mtm} .2013 .9$

\section{SUPPLEMENTARY MATERIAL}

The Supplementary Material for this article can be found online at https://www.frontiersin.org/articles/10.3389/fimmu.2018.00844/ full\#supplementary-material.

14. Xiao X, Li J, Samulski RJ. Production of high-titer recombinant adenoassociated virus vectors in the absence of helper adenovirus. J Virol (1998) 72:2224-32.

15. Li C, Xiao P, Gray SJ, Weinberg MS, Samulski RJ. Combination therapy utilizing shRNA knockdown and an optimized resistant transgene for rescue of diseases caused by misfolded proteins. Proc Natl Acad Sci U S A (2011) 108:14258-63. doi:10.1073/pnas.1109522108

16. Krishnan L, Gurnani K, Dicaire CJ, van Faassen H, Zafer A, Kirschning CJ, et al. Rapid clonal expansion and prolonged maintenance of memory CD8+ T cells of the effector (CD44highCD62Llow) and central (CD44highCD62Lhigh) phenotype by an archaeosome adjuvant independent of TLR2. J Immunol (2007) 178:2396-406. doi:10.4049/jimmunol.178.4.2396

17. Li C, Hirsch M, DiPrimio N, Asokan A, Goudy K, Tisch R, et al. CytotoxicT-lymphocyte-mediated elimination of target cells transduced with engineered adeno-associated virus type 2 vector in vivo. J Virol (2009) 83:6817-24. doi:10.1128/JVI.00278-09

18. He Y, Weinberg MS, Hirsch M, Johnson MC, Tisch R, Samulski RJ, et al. Kinetics of adeno-associated virus serotype 2 (AAV2) and AAV8 capsid antigen presentation in vivo are identical. Hum Gene Ther (2013) 24:545-53. doi:10.1089/hum.2013.065

19. Johnson JS, Samulski RJ. Enhancement of adeno-associated virus infection by mobilizing capsids into and out of the nucleolus. J Virol (2009) 83:2632-44. doi:10.1128/JVI.02309-08

20. Grieger JC, Snowdy S, Samulski RJ. Separate basic region motifs within the adeno-associated virus capsid proteins are essential for infectivity and assembly. J Virol (2006) 80:5199-210. doi:10.1128/JVI.02723-05

21. Girod A, Wobus CE, Zádori Z, Ried M, Leike K, Tijssen P, et al. The VP1 capsid protein of adeno-associated virus type 2 is carrying a phospholipase A2 domain required for virus infectivity. JGen Virol (2002) 83:973-8. doi:10.1099/0022-1317-83-5-973

22. Kronenberg S, Bottcher B, von der Lieth CW, Bleker S, Kleinschmidt JA. A conformational change in the adeno-associated virus type 2 capsid leads to the exposure of hidden VP1 N termini. J Virol (2005) 79:5296-303. doi:10.1128/JVI.79.9.5296-5303.2005

Conflict of Interest Statement: RS is the founder and a shareholder at Asklepios BioPharmaceutical and Bamboo Therapeutics, Inc. He holds patents that have been licensed by UNC to Asklepios BioPharmaceutical, for which he receives royalties. He has consulted for Baxter Healthcare and has received payment for speaking.

Copyright @ 2018 Pei, Earley, He, Chen, Hall, Samulski and Li. This is an open-access article distributed under the terms of the Creative Commons Attribution License (CC BY). The use, distribution or reproduction in other forums is permitted, provided the original author(s) and the copyright owner are credited and that the original publication in this journal is cited, in accordance with accepted academic practice. No use, distribution or reproduction is permitted which does not comply with these terms. 